\title{
Role of hepatocyte nuclear factor 4 alpha in cell proliferation and gemcitabine resistance in pancreatic adenocarcinoma
}

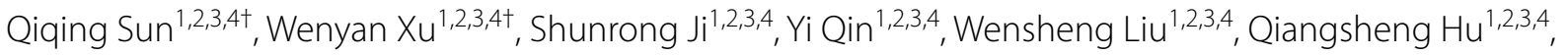
Zheng Zhang ${ }^{1,2,3,4}$, Mengqi Liu' ${ }^{1,2,3,4}$, Xianjun $\mathrm{Yu}^{1,2,3,4^{*}}$ and Xiaowu $\mathrm{Xu}^{1,2,3,4^{*}}$

\begin{abstract}
Background: Hepatocyte nuclear factor $4 a(H N F 4 a)$ is a tissue-specific transcription factor that regulates the expression of numerous genes in hepatocytes and pancreatic $\beta$ cells. HNF4a has been reported to affect cell proliferation and chemoresistance in several cancers. However, the role of HNF4a in pancreatic adenocarcinoma (PDAC) has not been studied extensively and remains unclear.
\end{abstract}

Methods: By utilizing immunohistochemical (IHC) staining, we measured the expression of HNF4a in PDAC tissues. By silencing HNF4a in PDAC cell lines, we assessed the impact of HNF4a on pancreatic cancer cell proliferation and gemcitabine sensitivity. We used CCK8 and colony formation assays to examine the effect of HNF4a on cell proliferation. A flow cytometry assay was used to assess cell apoptosis. The expression of gemcitabine-related genes was detected by quantitative real-time PCR (qRT-PCR) and Western blotting. IHC was utilized to assess the correlation between HNF4a and human equilibrative nucleoside transporter 1 (hENT1) expression in PDAC patients. Chromatin immunoprecipitation (ChIP) and dual-luciferase reporter assays were used to confirm that hENT1 is a target gene of HNF4a.

Results: Increased HNF4a expression was detected in PDAC tissues; patients with higher HNF4a expression displayed worse prognosis. To elucidate the function of HNF4a, we examined its role in pancreatic cancer cell proliferation, apoptosis and gemcitabine resistance. In HNF4a-silenced Capan-1 and MiaPaCa-2 cells, we observed decreased cell proliferation and increased sensitivity to gemcitabine compared to those of controls. The mechanism of HNF4a in gemcitabine-related chemosensitivity was then explored. In response to HNF4a silencing, the expression levels of gemcitabine-related proteins, hENT1 and deoxycytidine kinase (dCK) were significantly increased. Additionally, hENT1 was negatively correlated with HNF4a in PDAC tissue samples. Moreover, we identified hENT1 as a downstream target of HNF4a.

Conclusion: HNF4a is a prognostic marker for overall survival, is required for pancreatic cancer cell proliferation and promotes resistance to gemcitabine by downregulating hENT1. Therefore, targeting HNF4a might reverse gemcitabine resistance and provide novel treatment strategies for PDAC.

Keywords: Pancreatic adenocarcinoma, Hepatocyte nuclear factor 4a, Human equilibrative nucleoside transporter 1 , Proliferation, Gemcitabine resistance

\footnotetext{
*Correspondence: yuxianjun@fudanpci.org; xuxiaowu@fudanpci.org

${ }^{\dagger}$ Qiqing Sun and Wenyan Xu contributed equally to this article

3 Pancreatic Cancer Institute, Fudan University, Shanghai 200032, China

Full list of author information is available at the end of the article
} 


\section{Background}

Due to the lack of early diagnosis and limited therapy options, pancreatic ductal adenocarcinoma (PDAC) is still among the most lethal tumors and is the fourth leading cause of cancer-related deaths in the United States [1, 2]. Despite enormous progress in the treatment of many other tumor types in recent years, the main treatment for PDAC, other than surgery, is still based on gemcitabine alone or in combination with other chemotherapeutic agents [3]. However, the effect of gemcitabine and other chemotherapeutics remains unsatisfactory, partly due to the intrinsic chemoresistant characteristics of pancreatic cancer cells [4]. Therefore, exploration of potential targets to overcome gemcitabine resistance, which may highlight promising directions for developing novel therapeutic strategies, are urgently needed.

Hepatocyte nuclear factor $4 \alpha$ (HNF4 $\alpha$ or NR2A1), is an orphan nuclear receptor that is expressed in pancreas, liver, kidney and intestine [5, 6]. HNF4 $\alpha$ has been widely studied as an important regulator of hepatic functions, such as glycometabolism, fatty acid metabolism, and drug metabolism [7, 8]. Although there is abundant evidence that HNF $4 \alpha$ plays an important role in hepatic development, its role in the regulation of tumorigenesis and cancer development has been less widely studied. Darsigny et al. reported that HNF4 $\alpha$ was highly expressed in colorectal cancer tissue samples and promoted murine tumor development by targeting redox-related genes [9]. Conversely, another study suggested that low HNF4 $\alpha$ expression in colorectal cancer reduced the expression of the tumor suppressor gene CDX2 and that the lack of HNF4 $\alpha$ function promoted tumor progression [10]. Moreover, a recent study has suggested a role for HNF $4 \alpha$ in chemoresistance in gastric cancer, in which they reported that HNF $4 \alpha$ may enhance multidrug resistance by regulating cell apoptosis and expression of B-cell lymphoma 2 (Bcl2) [11]. However, the role of HNF4 $\alpha$ in PDAC development has not been reported widely and needs further investigation.

As a highly hydrophilic chemical, gemcitabine requires integral membrane transporters to function as a mediator for drug intake. It has been reported that a membrane transporter protein, human equilibrative nucleoside transporter 1 (hENT1), is responsible for the majority of gemcitabine uptake [12,13]. Once it is in the cytoplasm, gemcitabine undergoes rate-limiting steps, as the monophosphate is phosphorylated by deoxycytidine kinase (dCK) to generate its active metabolites, gemcitabine diphosphate and gemcitabine triphosphate $[14,15]$. The most important mechanism of gemcitabine cytotoxicity is inhibition of DNA synthesis, in which ribonucleotide reductase (RR) plays an important role [16-18]. First, the diphosphate form of gemcitabine can inactivate
RR subunit M1 (RRM1) and RR subunit M2 (RRM2). Additionally, gemcitabine triphosphate can embed into DNA chains, which causes termination of DNA synthesis, resulting in cancer cell apoptosis [19]. In brief, hENT1, dCK and RR are central proteins that contribute to gemcitabine cytotoxicity, and many previous studies have confirmed that their expression correlates with gemcitabine efficacy and patient prognosis [13, 20,21].

In the present study, we explored the function of HNF $4 \alpha$ and its underlying mechanism in PDAC. Our study provided novel findings, including that HNF4 $\alpha$ silencing resulted in decreased cell proliferation and increased gemcitabine sensitivity, which was partly due to transcriptional repression of hENT1. Collectively, our present study uncovered novel predictive and treatment targets that show promise for improving overall survival for PDAC.

\section{Methods}

\section{Clinical samples and cell lines}

Clinical tissue samples were obtained from patients who were diagnosed at the Department of Pancreatic Surgery at Fudan University Shanghai Cancer Center (FUSCC) in 2012. We obtained informed consent from the patients and were approved by the Institutional Research Ethics Committee of FUSCC. The clinical characteristics of the samples are summarized in Table 1 . The human pancreatic cancer cell lines Capan-1 and MiaPaCa-2 were purchased from the American Type Culture Collection (ATCC, USA) and were cultured according to standard ATCC protocols. In brief, PANC-1 cells were cultured in Iscove's modified Dulbecco's medium containing $20 \%$ fetal bovine serum (FBS), $100 \mathrm{U} / \mathrm{mL}$ penicillin and $0.1 \mathrm{mg} / \mathrm{mL}$ streptomycin. MiaPaCa-2 cells were maintained in Dulbecco's modified Eagle's medium with 10\% FBS and an additional $2.5 \%$ horse serum. These cells were cultured at $37^{\circ} \mathrm{C}$ in a humidified incubator with $5 \% \mathrm{CO}_{2}$.

\section{Immunohistochemical (IHC) staining}

IHC staining of paraffin-embedded tissues with antibodies against HNF4 $\alpha$ (1:200; CST, USA), hENT1 (1:200; Proteintech, USA) and dCK (1:800; Abcam, UK) were performed using standard procedures as described previously [22-24]. The evaluation criterion considered both the percentage of stained positive cells $(0,<5 \% ; 1,5-25 \%$; $2,25-50 \% ; 3,50-75 \% ; 4,>75 \%)$ and the staining intensity ( 0 , negative; 1 , weakly positive; 2 , moderately positive; 3 , strongly positive). The total scores were calculated as the product of staining frequency and intensity. The expression levels were classified as follows: negative $(0-3,-)$; weakly positive $(4,+)$; moderately positive $(6,++)$; and strongly positive $(>6,+++)$. The patients were divided 
Table 1 Relationship between HNF4a expression and clinicopathological features of pancreatic cancer

\begin{tabular}{|c|c|c|c|c|}
\hline \multirow[t]{2}{*}{ Variables } & \multirow{2}{*}{$\begin{array}{l}\text { Patient } \\
\text { number } \\
\text { (n) }\end{array}$} & \multicolumn{3}{|c|}{$\begin{array}{l}\text { HNF4a expression in tumor tissue } \\
\text { of pancreatic cancer }\end{array}$} \\
\hline & & Low $(n=41)$ & High $(n=64)$ & $P$ value \\
\hline \multicolumn{4}{|l|}{ Gender } & 0.9321 \\
\hline Male & 62 & 24 & 38 & \\
\hline Female & 43 & 17 & 26 & \\
\hline \multicolumn{4}{|l|}{ Age (years) } & 0.2434 \\
\hline$\leq 60$ & 54 & 24 & 30 & \\
\hline$>60$ & 51 & 17 & 34 & \\
\hline \multicolumn{4}{|l|}{ TNM stage } & 0.8487 \\
\hline I, Ila & 55 & 21 & 34 & \\
\hline IIb, III, IV & 50 & 20 & 30 & \\
\hline \multicolumn{4}{|c|}{ Tumor size (cm) } & 0.2764 \\
\hline$\leq 4$ & 73 & 26 & 47 & \\
\hline$>4$ & 32 & 15 & 17 & \\
\hline \multicolumn{4}{|c|}{ Histological grade } & 0.1917 \\
\hline Grade 1, 2 & 62 & 21 & 41 & \\
\hline Grade 3 & 43 & 20 & 23 & \\
\hline \multicolumn{4}{|c|}{ Lymph node status } & 0.8625 \\
\hline Negative & 60 & 23 & 37 & \\
\hline Positive & 45 & 18 & 27 & \\
\hline \multicolumn{4}{|c|}{ Vascular emboli } & 0.6061 \\
\hline Negative & 87 & 33 & 54 & \\
\hline Positive & 18 & 8 & 10 & \\
\hline
\end{tabular}

into two groups $(\mp$, low expression and $++/+++$, high expression) for survival analyses.

\section{Plasmids}

The 21 base pair (bp) targets against HNF4 $\alpha$ were TCA GGGTCTGAGCCCTATAAG and CCATCACCAAGC AGGAAGTTA. shRNA oligos were synthesized and ligated into an LKO.1 TRC cloning vector (Addgene, USA) according to the standard procedures provided by Addgene [25], and HNF4 $\alpha$ silencing lentivirus constructs were designated pLKO.1-shHNF4 $\alpha-\mathrm{A}$ and pLKO.1shHNF4 $\alpha$-B. pLKO.1-shHNF4 $\alpha$ was cotransfected with psPAX2 and pMD2.G into HEK-293T cells at a ratio of 4:3:1 for the production of lentiviral particles. pLKO.1shscramble (Addgene, USA) was used as the control plasmid.

\section{Quantitative real-time PCR (qRT-PCR)}

TRIzol reagent (Invitrogen, USA) was used to extract total RNA. Reverse transcription was performed using a TaKaRa PrimeScript RT reagent kit (TaKaRa, Japan) to obtain cDNA. The expression of candidate genes was determined using an ABI 7900HT Real-time PCR system
Table 2 Primer sequences used in this study

\begin{tabular}{|c|c|}
\hline HNF4a forward & 5'-GGTGTTCAAGGACGTGCTGCTCC-3' \\
\hline HNF4a reverse & 5'-AGTCCTCCAAGCTCACCTGCACC-3' \\
\hline hENT1 forward & 5'-CTCCAACTCTCAGCCCACCAATGA-3' \\
\hline hENT1 reverse & 5'-GAAGTAACGTTCCCAGGTGCTGC-3' \\
\hline$d C K$ forward & 5'-CAAGACTGGCATGACTGGATGAA-3' \\
\hline$d C K$ reverse & 5'-GGCACCTCTTGAAGATAATCGAAG-3' \\
\hline RRM1 forward & $5^{\prime}$-TGGAGTACACCAGCAAAGATGAGG-3' \\
\hline RRM1 reverse & 5'-GGCGATGGCGTTTATTTGATAGGC-3' \\
\hline$\beta$-actin forward & 5'-AGAGCTACGAGCTGCCTGAC-3' \\
\hline$\beta$-actin reverse & 5'-AGCACTGTGTTGGCGTACAG-3' \\
\hline ChIP forward 1 & 5'-TGCCTCACTGGCCTCTCCCTAGTC-3' \\
\hline ChIP reverse 1 & 5'-CACCACCCTATATGGGACCGTGGC-3' \\
\hline ChIP forward 2 & 5'-TTTGAATGTGCCCCGGCGGGAGA-3' \\
\hline ChIP reverse 2 & 5'-TCCCTGGCCCGTGCGCGCCACGT-3' \\
\hline
\end{tabular}

(Applied Biosystems, USA). The primer sequences used in this study are presented in Table 2.

\section{Western blotting analysis}

Western blotting was conducted as described in a previous study [24]. The antibodies used in the present study were $\beta$-actin (1:5000; Proteintech, USA), HNF4 $\alpha$ (1:1000; CST, USA), p21 (1:1000; Proteintech, USA), p27 (1:1000; Proteintech, USA), Caspase- 9 (1:1000; CST, USA), PARP (1:1000; CST, USA), ENT1 (1:1000; Proteintech, USA), DCK (1:1000; Abcam, UK), and RRM1 (1:1000; Proteintech, USA).

\section{CCK-8 assay}

Cell proliferation and cytotoxicity was measured via cell viability using a Cell Counting Kit-8 (Dojindo, Japan) and was conducted as previously described [26]. The concentration of gemcitabine that inhibited cell viability by $50 \%$ (IC50 value) was calculated from a nonlinear least squares curve that was fit to the dose-response curves.

\section{Colony formation assay}

Cells (500/well) were seeded and cultured in six-well plates, and cells were fixed with $4 \%$ paraformaldehyde followed by $0.1 \%$ crystal violet (Sigma, USA) staining after 2 weeks of cultivation. Colonies were quantified under a light microscope.

\section{Cell apoptosis analysis}

Cells were stained with fluorescein isothiocyanate-conjugated annexin $\mathrm{V}$ and propidium iodide (BD, La Jolla, CA, USA) according to the manufacturer's instructions, and the percentage of apoptotic cells was measured using a FACSCalibur flow cytometer. 


\section{Promoter activity assessment by dual-luciferase assay}

The hENT1 promoter region, spanning from -3000 to 300 of the transcription starting site, was amplified from genomic DNA and cloned into a pGL3-Basic vector. HEK-293T cells were seeded on 96-well culture plates and transfected with the pGL3 constructs as well as Renilla luciferase expression vectors using Lipofectamine 2000 (Invitrogen, USA). Both firefly and Renilla luciferase activities were assayed using a dual-luciferase system (Promega, USA) that complied with the manufacturer's protocol.

\section{Chromatin immunoprecipitation (ChIP) assay}

ChIP was conducted according to the instructions of a Magna ChIP $^{\mathrm{TM}}$ A/G Chromatin Immunoprecipitation Kit (Merck Millipore Corporation). The nuclear DNA extracts were amplified using two pairs of primers that spanned the hENT1 promoter region (Table 2).

\section{Statistics}

All data are presented as the mean $\pm \mathrm{SD}$, and experiments were repeated at least three times. The data were analyzed using SPSS 22.0 software (Abbott Laboratories, USA). Student's test and one-way ANOVA were used to analyze the data between groups. A Chi square test was performed to analyze the relationships between HNF4 $\alpha$ expression and clinicopathologic characteristics. Log-rank test and Cox regression were used in survival analysis for patients with PDAC. Spearman correlation analysis was used to determine the correlation between the HNF $4 \alpha$ and hENT1 expression levels. $P<0.05$ was considered statistically significant.

\section{Results}

HNF4a expression is positively correlated with PDAC prognosis

We first used IHC to examine the HNF4 $\alpha$ levels in 30 paired-patient samples of PDAC and adjacent normal tissues (Fig. 1a, b). Then, we further validated the IHC results by examining HNF4 $\alpha$ expression in tissue microarrays containing 105 pairs of patient samples (Fig. 1c, Additional file 1: Table S1). The IHC score showed that HNF4 $\alpha$ was expressed at higher levels in PDAC tissues than in control tissues (Additional file 1: Table S1). Moreover, the log-rank test revealed an obvious correlation between high HNF4 $\alpha$ expression and poor prognosis of PDAC patients $(P=0.0281$; Fig. $1 \mathrm{~d})$. Pancreatic cancer patients with higher HNF4 $\alpha$ expression exhibited significantly shorter overall survival compared to patients with low HNF4 $\alpha$ expression, with a median survival time of 11.015 months vs. 17.1 months. Furthermore, according to Cox regression analysis, HNF4 $\alpha$ was an independent prognostic marker of PDAC (Table 3). Finally, we investigated the levels of $H N F 4 \alpha$ protein in various PDAC cell lines (Fig. 1e). HNF4 $\alpha$ was highly expressed in PANC-1, Capan-1 and MiaPaCa-2 cell lines. We ultimately chose Capan-1 and MiaPaCa- 2 cell lines for further investigation.

\section{HNF4a promotes proliferation of pancreatic cancer cells} Based on the vital role of HNF4 $\alpha$ in PDAC prognosis, we hypothesized that HNF4 $\alpha$ might promote pancreatic cancer cell proliferation. Stable HNF4 $\alpha$-silencing cell lines were obtained by infecting Capan-1 and Mia$\mathrm{PaCa}-2$ cells with lentiviral particles and by subsequently using puromycin selection. The knock-down efficiency was verified by qRT-PCR and Western blotting (Fig. 2a, b). The CCK- 8 assay showed that HNF4 $\alpha$ silencing significantly decreased cell viability in Capan-1 and MiaPaCa-2 cells (Fig. 2c). Subsequent colony formation assay results demonstrated that HNF $4 \alpha$ abrogation decreased colony formation capacity in Capan-1 and MiaPaCa-2 cells (Fig. 2d). Further, Western blotting analysis showed increases in p21 and p27 expression in HNF4 $\alpha$-silenced cell lines, which indicated an activated tumor-suppressive mechanism of HNF4 $\alpha$ knockdown (Fig. 2e) [27]. In summary, HNF4 $\alpha$ may play oncogenic roles and enhances pancreatic cancer cell proliferation.

\section{Silencing of HNF4a increases pancreatic cancer cell sensitivity to gemcitabine}

Since evidence has suggested that loss of $\mathrm{p} 21$ or $\mathrm{p} 27$ function may contribute to cancer cell chemoresistance [27, 28], we subsequently explored the relationship between HNF $4 \alpha$ and gemcitabine sensitivity. We first used a viability assay to analyze the effects of HNF $4 \alpha$ abrogation on gemcitabine-treated cells. The results suggested that HNF4 $\alpha$ silencing dramatically decreased the IC50 values of gemcitabine both in Capan-1 and MiaPaCa- 2 cells at $48 \mathrm{~h}$ (Fig. 3a). Gemcitabine exerts its antitumor activity mainly by inducing cell apoptosis [29], and we then investigated gemcitabine-induced apoptosis. The apoptotic rate induced by gemcitabine was further enhanced in HNF4 $\alpha$-silenced cell lines (Fig. 3b, c), with increased expression of apoptosis-related proteins such as cleaved poly (ADP-ribose) polymerase-1 (PARP1) and cleaved caspase-9 (Fig. 3d) [30, 31].

\section{HNF4a is negatively correlated with hENT1 expression}

We next examined whether HNF4 $\alpha$ silencing could regulate the expression of gemcitabine metabolismrelated proteins. Numerous studies have indicated that hENT1, dCK and RRM1 are key proteins for gemcitabine resistance in pancreatic cancer (Fig. 4a) [19, $20,32,33]$. The subsequent qRT-PCR and Western 


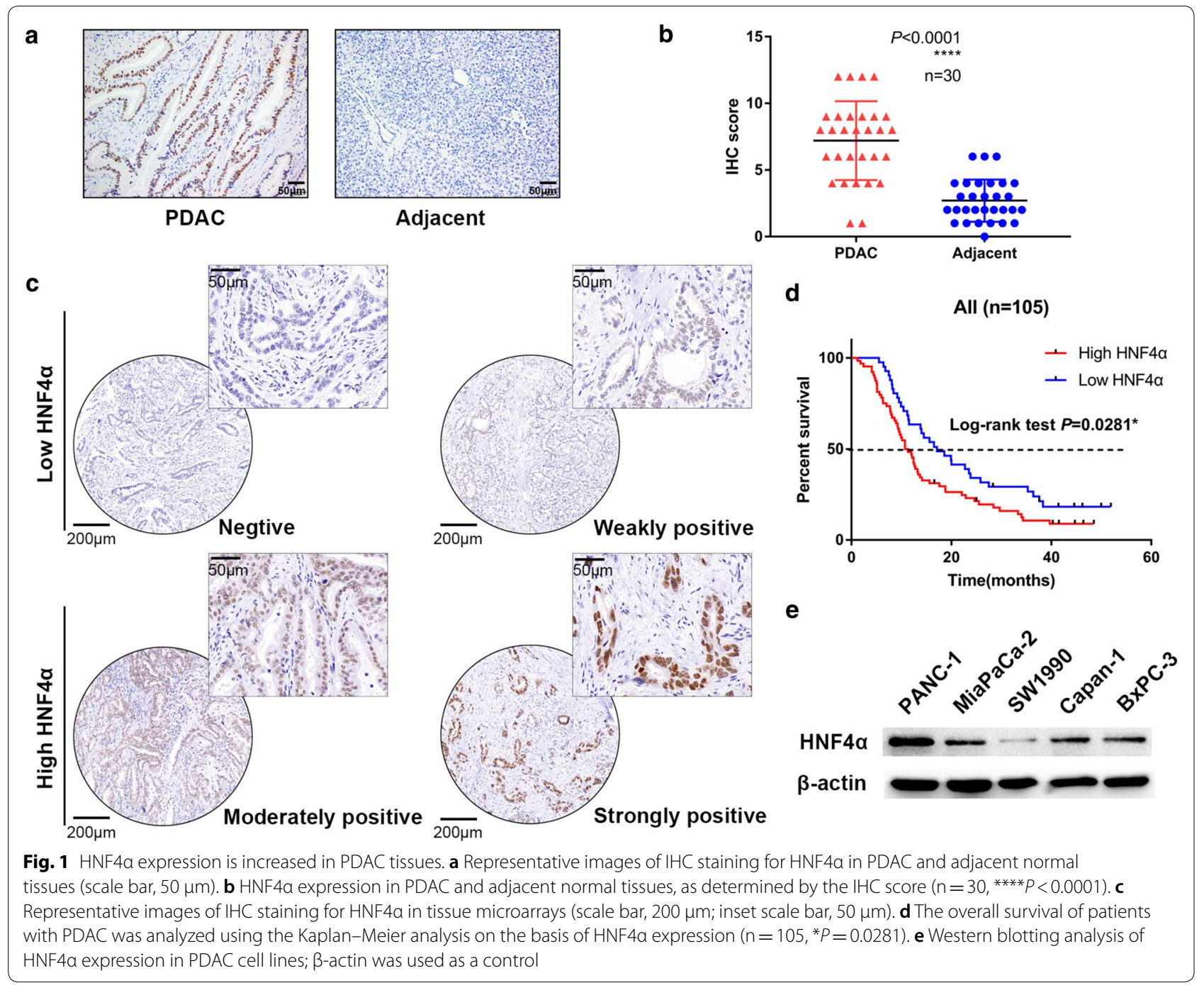

blotting results exhibited upregulated expression of hENT1 and dCK after deletion of HNF4 $\alpha$ in Capan-1 and MiaPaCa-2 cell lines, whereas RRM1 presented no obvious change at both the transcriptional and protein levels (Fig. 4b, c). To further validate the results of the in vitro experiments, we examined the correlation between hENT1, dCK and HNF4 $\alpha$ in PDAC patients. We found that hENT1 expression negatively correlated with HNF4 $\alpha$ levels in PDAC patient tissue samples (Fig. 4d, e). Moreover, consistent with the results of previous studies, the IHC staining of tissue microarrays indicated that higher hENT1 expression indicated a better prognosis in PDAC (Additional file 2: Figure S1, $\mathrm{a}, \mathrm{b}, \mathrm{n}=105, P=0.0208)$. However, Spearman's correlation showed no obvious correlation between $\mathrm{dCK}$ and HNF4 $\alpha$ levels in PDAC patient tissue samples (Additional file 3: Figure $\mathrm{S} 2, \mathrm{n}=30, P=0.4281$ ).

\section{hENT1 is a downstream target of HNF4a}

Based on these results, we hypothesized that hENT1 may be a target of and regulated by HNF4 $\alpha$. In the promoter region of hENT1, one possible HNF4 $\alpha$ binding element exists (Fig. 5a). Thus, we conducted a ChIP assay to determine whether HNF4 $\alpha$ could occupy the consensus HNF $4 \alpha$ binding element. ChIP results demonstrated that HNF4 $\alpha$ could bind the HNF4 $\alpha$ binding element in the promoter region of hENT1. Moreover, ChIP results also demonstrated that HNF4 $\alpha$ could occupy the region from -63 to +99 , indicating that other regulatory mechanisms may exist (Fig. 5b). Subsequent luciferase assays demonstrated that manipulation of HNF4 $\alpha$ expression inhibited hENT1 promoter activity in a dose-dependent manner (Fig. 5c). This phenomenon was further confirmed by the mutation of hENT1 binding sites; when the sequence was mutated from AGCTGAGAGGACA into AGCTGAGACCAAA to generate a mutant, HNF4 $\alpha$ lost 
Table 3 Univariate and multivariate Cox regression of overall survival for patients with PDAC

\begin{tabular}{|c|c|c|c|c|c|c|}
\hline \multirow[t]{2}{*}{ Characteristics } & \multicolumn{3}{|c|}{ Univariate } & \multicolumn{3}{|c|}{ Multivariate } \\
\hline & HR & $95 \% \mathrm{Cl}$ & $P$ value & HR & $95 \% \mathrm{Cl}$ & $P$ value \\
\hline \multicolumn{7}{|l|}{ Age (years) } \\
\hline$>60$ & 1.195 & 0.788 to 1.812 & 0.402 & & - & \\
\hline \multicolumn{7}{|l|}{$\leq 60$} \\
\hline \multicolumn{7}{|l|}{ Gender } \\
\hline Male & 0.842 & 0.550 to 1.290 & 0.430 & & - & \\
\hline \multicolumn{7}{|l|}{ Female } \\
\hline \multicolumn{7}{|l|}{ TNM stage } \\
\hline IIb, III, IV & 1.722 & 1.133 to 2.619 & $0.011^{*}$ & & - & \\
\hline \multicolumn{7}{|l|}{ I, lla } \\
\hline \multicolumn{7}{|l|}{ Tumor size (cm) } \\
\hline$\geq 4.0$ & 1.809 & 1.148 to 2.851 & $0.011^{*}$ & 1.919 & 1.210 to 3.041 & $0.006^{* *}$ \\
\hline \multicolumn{7}{|l|}{$<4.0$} \\
\hline \multicolumn{7}{|l|}{ Histological grade } \\
\hline Grade 3 & 1.200 & 0.782 to 1.841 & 0.403 & & - & \\
\hline \multicolumn{7}{|l|}{ Grade 1,2 } \\
\hline \multicolumn{7}{|c|}{ Lymph node status } \\
\hline Positive & 1.826 & 1.201 to 2.778 & $0.005^{* *}$ & 1.887 & 1.237 to 1.049 & $0.003^{* *}$ \\
\hline \multicolumn{7}{|l|}{ Negative } \\
\hline \multicolumn{7}{|l|}{ Vascular emboli } \\
\hline Positive & 1.012 & 0.571 to 1.791 & 0.968 & & - & \\
\hline \multicolumn{7}{|l|}{ Negative } \\
\hline \multicolumn{7}{|l|}{ HNF4a expression } \\
\hline High & 1.560 & 1.012 to 2.404 & $0.044^{*}$ & 1.616 & 1.049 to 2.491 & $0.030^{*}$ \\
\hline Low & & & & & & \\
\hline
\end{tabular}

its repressive function for the mutated luciferase construct (Fig. 5d).

In conclusion, our results demonstrated that HNF4 $\alpha$ is a novel predictive marker for overall survival in PDAC. In vitro cell line studies demonstrated that HNF4 $\alpha$ promoted proliferation and gemcitabine resistance to PDAC cancer cell lines. Mechanistically, HNF4 $\alpha$ suppressed the expression of hENT1, which was responsible for gemcitabine uptake and was correlated with gemcitabine resistance (Fig. 6).

\section{Discussion}

Considering the significant role of gemcitabine in adjuvant therapy and the treatment of patients with unresectable PDAC, elucidating the underlying mechanisms of gemcitabine resistance could improve treatment response [19, 34]. In the present study, we investigated the role of HNF4 $\alpha$ in PDAC. Our results indicated that HNF4 $\alpha$ enhances pancreatic cancer cell proliferation and promotes gemcitabine resistance by downregulating the transcription of hENT1. Moreover, HNF4 $\alpha$ levels constitute an important prognostic factor for patients with PDAC.
HNF4 $\alpha$ is a highly conserved nuclear receptor belonging to the nuclear receptor (NR) superfamily [6]. As the third class of nuclear receptors, orphan receptors are characterized by a lack of endogenous ligands [35]. Despite a well-known role in maintaining cellular homeostasis, NRs have also been found to participate in cancer proliferation and progression $[36,37]$. NRs play diverse roles in the oncogenesis and progression of many cancers. Estrogen receptor $\alpha$ and androgen receptor are widely recognized as oncogenes, while other NRs, such as proliferator-activated receptor $\gamma$, may have inhibitory functions on cancer proliferation [38-40]. Moreover, certain NRs may have opposing functions in different cancer types. For example, the orphan nuclear receptor NR4A1 (Nur77) played a role in suppressing hepatocellular carcinoma by inducing a glycolysis to gluconeogenesis switch, whereas it facilitated cancer cell proliferation via the ROS/endoplasmic reticulum stress pathways in pancreatic cancer [41, 42]. NRs also play an important role in regulating drug metabolism in cancer therapy. Holbeck et al. reported that cancer sensitivity to microtubuledisrupting drugs, such as derivatives of vinblastine, colchicines, and Taxol, was increased in cells expressing low 

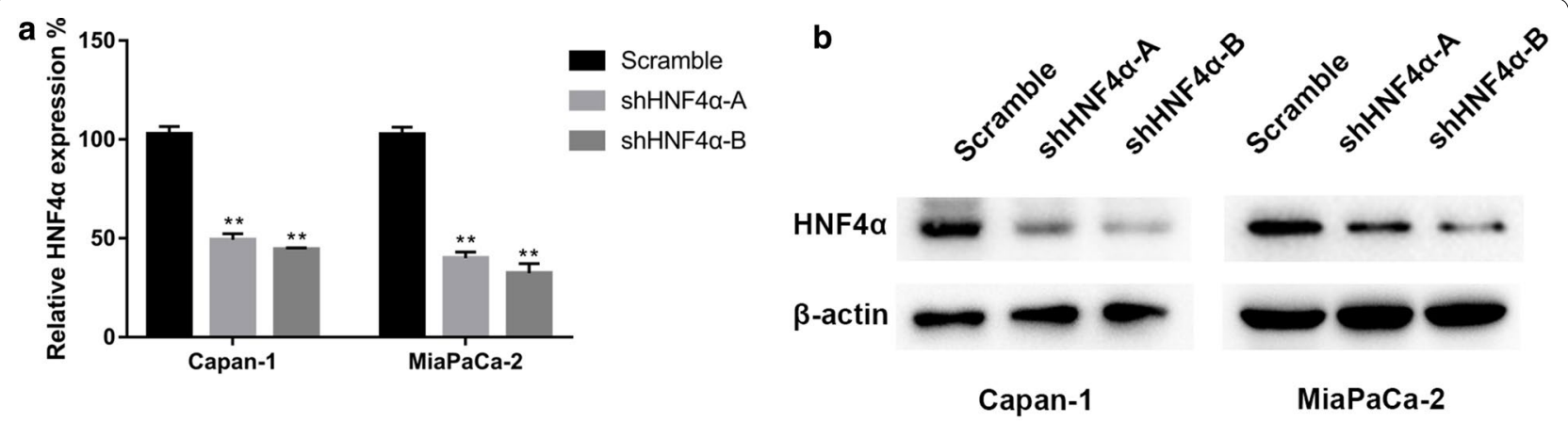

Capan-1

C

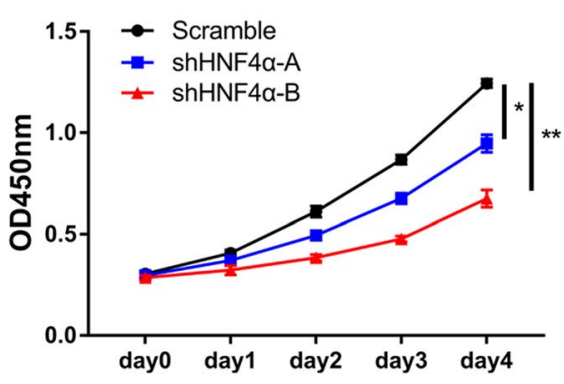

MiaPaCa-2

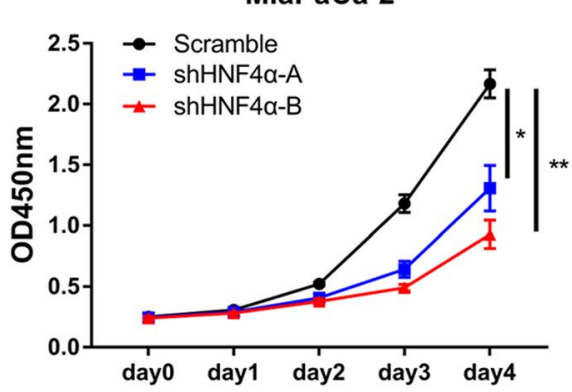

d

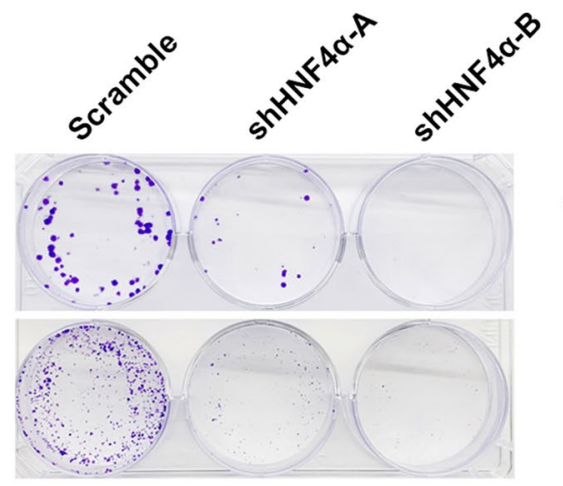

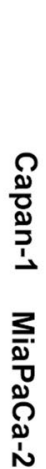

e

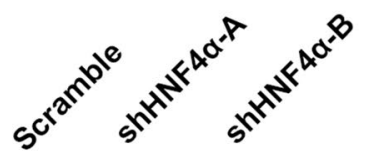

p21

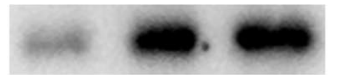

p27

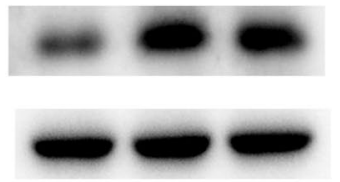

Capan-1
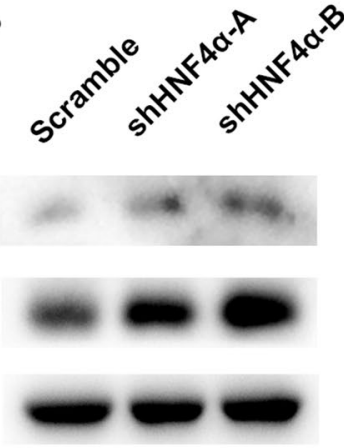

MiaPaCa-2

Fig. 2 Specific silencing of HNF4a decreased the proliferation of pancreatic cancer cells. a Analysis of relative gene expression data of HNF4a using real-time quantitative PCR and the 2- $\triangle \triangle C T$ method. $\mathbf{b}$ Analysis of protein expression of HNF4a using western blotting assay. $\mathbf{c} C C K-8$ assay was used to test the proliferation of PDAC cells transfected with the HNF4a shRNA. $\mathbf{d}$ Colony formation assay was conducted to confirm the influence of abrogation of HNF4a on pancreatic cancer cell lines; $\mathbf{f}$ The p21 and p27 expression levels in HNF4a-silencing pancreatic cancer cell lines were analyzed comparing to the controls by western blotting. *Represented significant differences $(P<0.05$, compared with group control); **Represented with significant differences $(P<0.01$, compared with group control)

levels of NR2F2 based on NR expression profiling, while the high levels of the orphan receptor tailless were correlated with $9 \alpha$-fluoroprednisolone sensitivity [43].

Considering the significant roles of NRs in cancer development and the high expression of HNF4 $\alpha$ in gastrointestinal cancers, such as colorectal carcinoma [43], we investigated whether $\mathrm{HNF} 4 \alpha$ regulated the biological behaviors of pancreatic cancer. We found that abrogation of $\mathrm{HNF} 4 \alpha$ expression inhibited pancreatic cancer cell proliferation and induced cell apoptosis, with increased expression of the cyclin-dependent protein kinase inhibitors $\mathrm{p} 21$ and $\mathrm{p} 27$. HNF4 $\alpha$ has been reported to inhibit hepatocyte proliferation via several proposed mechanisms, including direct inhibition of mitogenic genes, regulation of the c-Myc and cyclin pathways, and an HNF4 $\alpha$-driven miRNA feedback loop [36]. Our results indicated that HNF4 $\alpha$ may promote cell proliferation via the cyclin pathways in pancreatic cancer, but further 


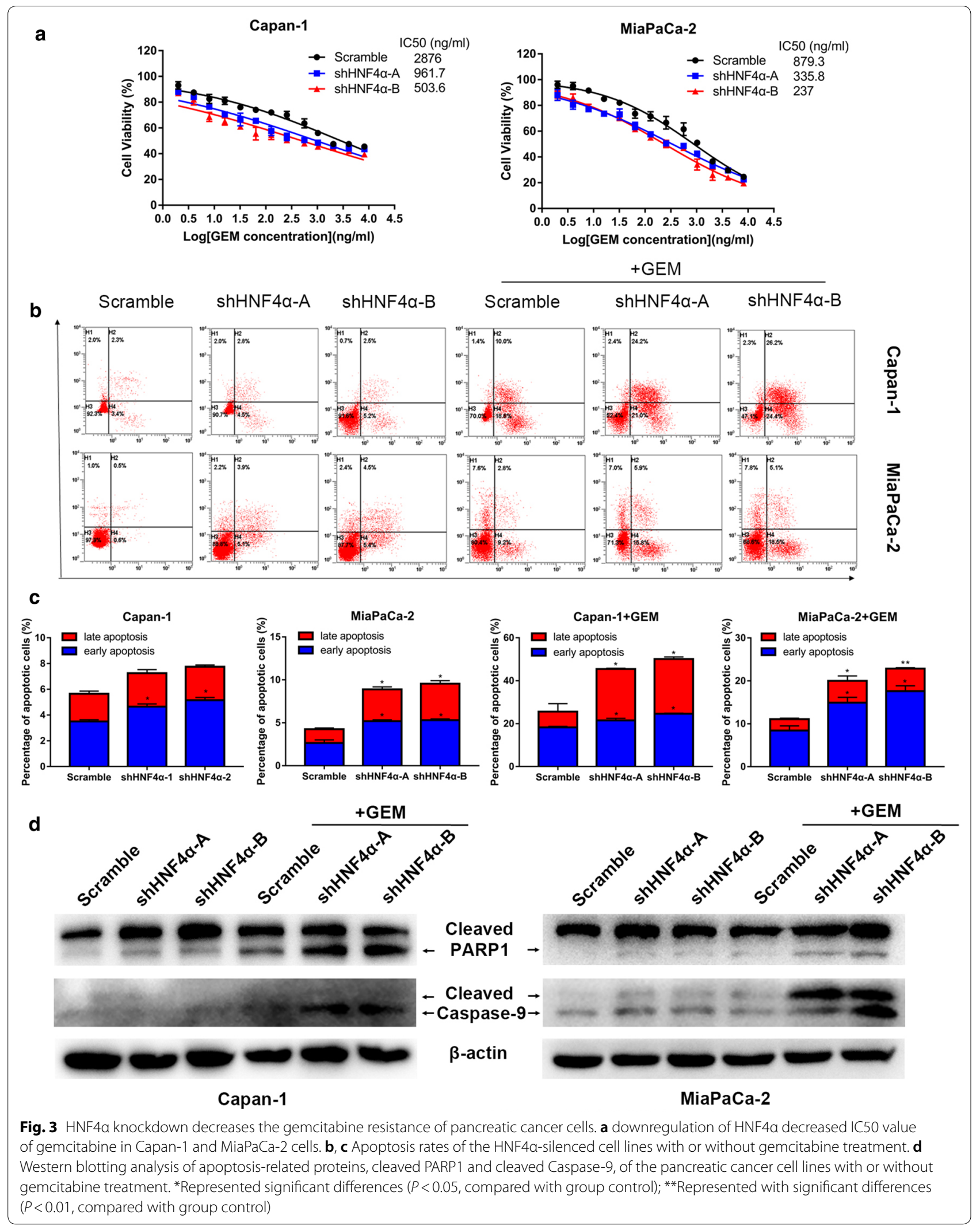




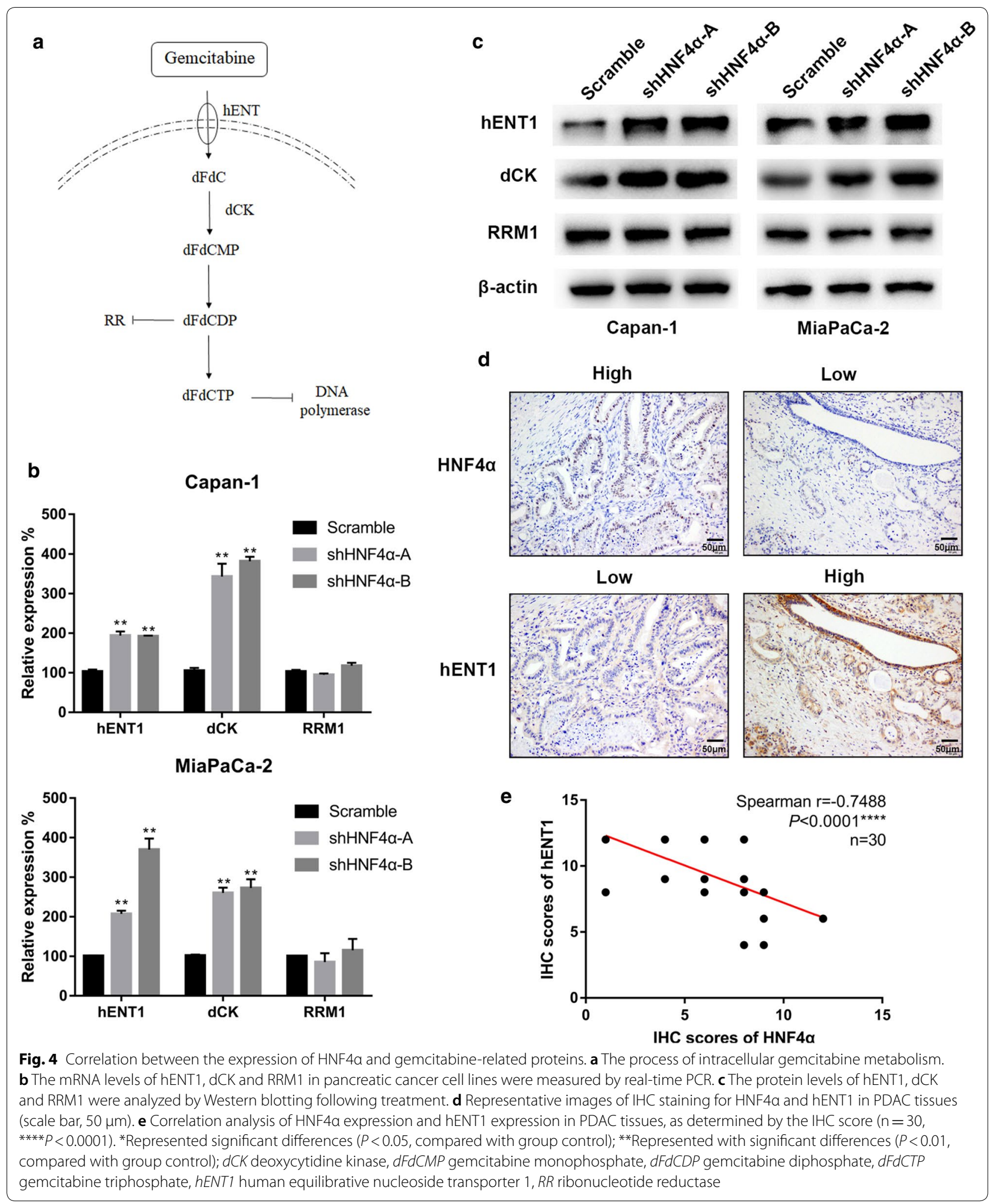


a

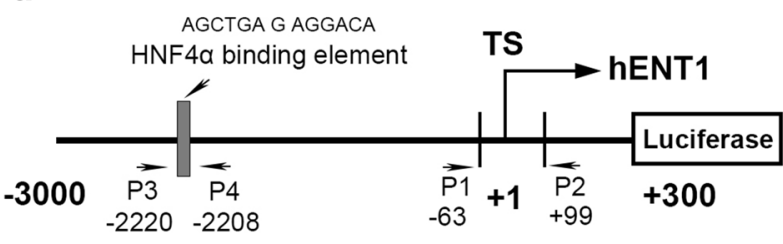

b HNF4 $\alpha$ consensus

b binding site

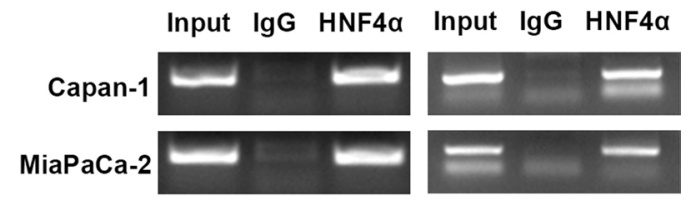

c

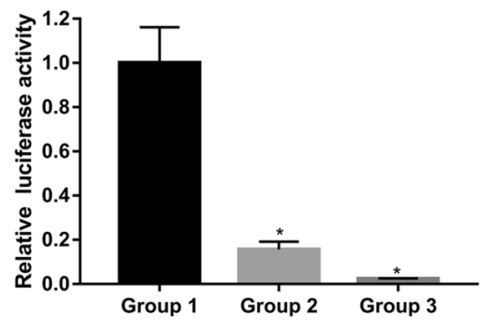

PGL3-hENT1 200ng

HNF4 $\alpha$

Renilla

\section{0ng 200ng}

200ng 500ng

10ng 10ng d

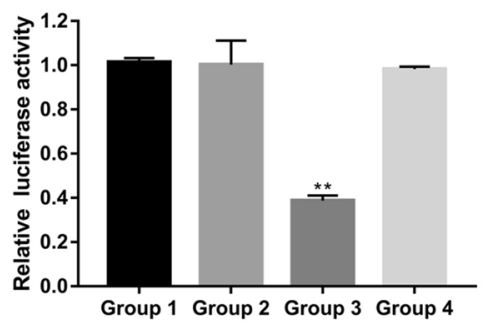

pGL3-hENT1 200ng - 200ng -

HNF4 $\alpha$ - - 200ng 200ng

pGL3-hENT1 1MUT - 200ng - 200ng

Renilla 10ng 10ng 10ng 10ng

Fig. 5 HNF4a was involved in the hENT1 transcriptional expression in PDAC. a The position of the HNF4a binding sites in the hENT1 promoter. b HNF4a occupies the binding sites of the hENT1 promoter region in Capan-1 and MiaPaCa cells, as measured by ChIP assay. c HNF4a affected hENT1 promoter activity in HEK-293T cells. d HNF4a didn't affect the mutated hENT1 promoter activity in HEK-293T cells

investigation is needed to confirm this hypothesis. Moreover, in vivo experiments are required to support the oncogenic role of HNF4 $\alpha$ in PDAC in the future.

The mechanisms of chemoresistance of PDAC may involve the hypoxic tumor microenvironment and deficient vascularization, remodeled metabolism, the capacity for epithelial-mesenchymal transition and altered key signaling pathways, such as the PI3 K/Akt and NF-KB signaling pathways $[19,44-49]$. As a key transporter for the cellular uptake of gemcitabine, the downregulation of hENT1 is one of the recognized mechanisms of gemcitabine resistance [19]. Although the role of hENT1 as a predictive molecule for the response to gemcitabinebased therapy has been confirmed by many studies using adjuvant treatment and studying patients with unresectable tumors, in which higher expression of hENT1 was correlated with better survival, the molecular mechanisms of hENT1 regulation have seldom been described [20, 50-52]. Pandolfi et al. reported that high levels of glucose could upregulate the formation of hCHOP-C/ EBPa, a complex that represses hENT1 expression [53]. A. Hesler et al. found that hENT1 could be negatively regulated by the matricellular protein cysteine-rich angiogenic inducer 61 , but the underlying mechanism requires further exploration [54]. Hu et al. proposed the role of FBW7 in the regulation of hENT1 at the protein level, possibly via the inhibition of the lysosome degradation by hENT1 [24].

Our study revealed that hENT1 could be regulated by $\mathrm{HNF} 4 \alpha$ at the transcriptional level. HNF4 $\alpha$ mainly binds to a 6-bp repeat (AGGTCA) with a nucleotide spacer called direct repeat 1 (DR1) $[55,56]$. Our results showed that HNF4 $\alpha$ recognized the consensus binding site located on the promoter region of hENT1 and activated downstream transcription. Moreover, a relatively nonconserved region with abundant CPG dinucleotide could also be recognized by HNF4 $\alpha$, which indicates that HNF4 $\alpha$ might participate in the methylation process of hENT1. A previous study demonstrated DNA methylation-independent hENT1 downregulation, which correlated with acquired gemcitabine resistance in cancer cells [57]. Our study supports the need for further investigation of the underlying mechanism of methylationdependent hENT1 regulation. Moreover, after binding to DNA in the form of a homologous dimer, how HNF4 $\alpha$ recruits transcriptional corepressors and accessory proteins to regulate hENT1 is unclear. HNF4 $\alpha$ has been reported by previous studies to interact with chromatin modifiers, such as PRMT1 and BMI1 [58, 59], suggesting a potential role of HNF4 $\alpha$ in chromatin methylation. Furthermore, in addition to the expression level of hENT1, hENT1 transporter activity is another important factor 


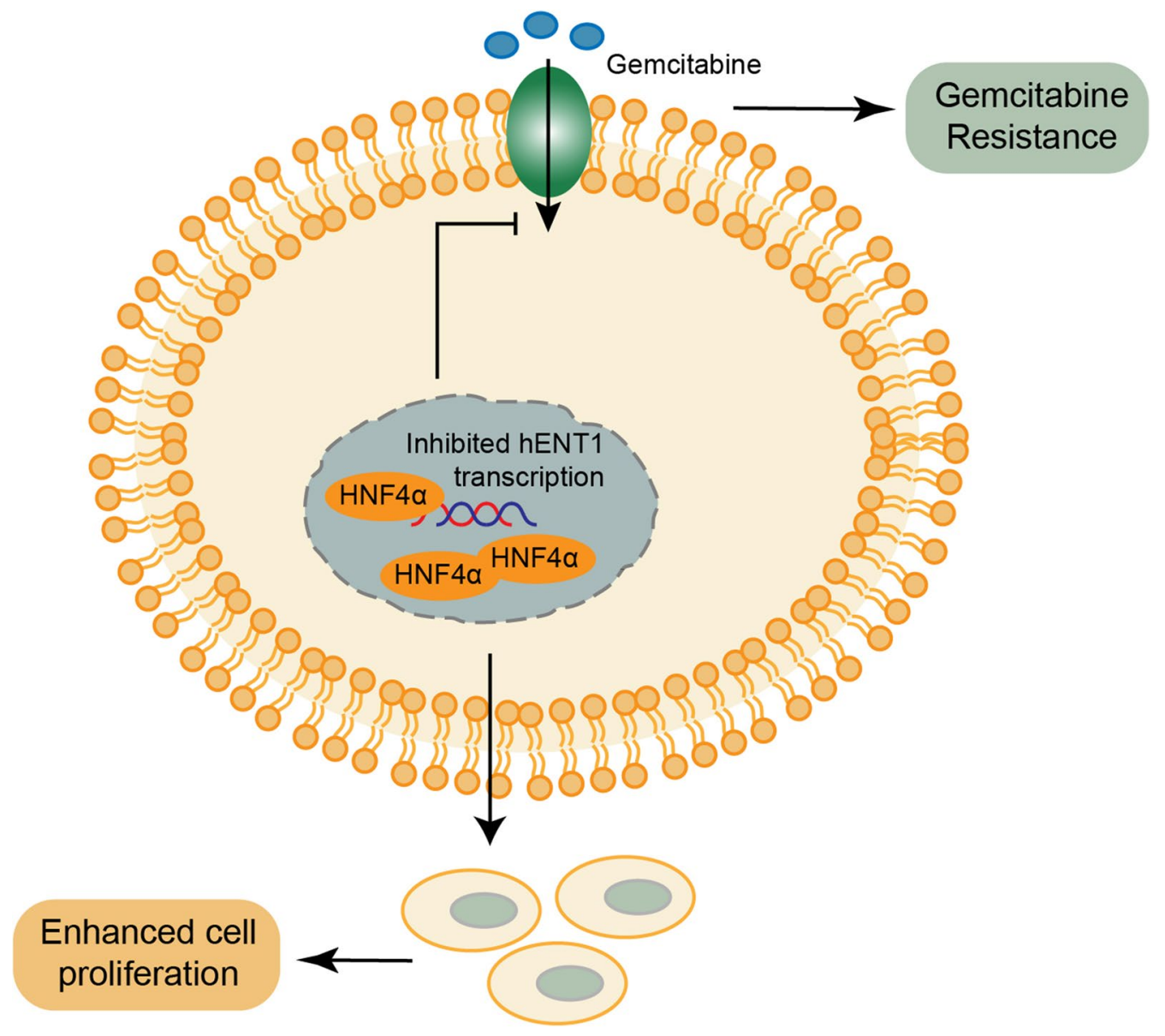

Fig. 6 Schematic representation of the model. The model indicates the mechanism of HNF4a-mediated regulation of gemcitabine metabolism via hENT1 in pancreatic cancer cells and the role of HNF4a in cancer cell proliferation

that affecting the uptake of nucleoside analogs. A study has reported that although the mRNA level of hENT1 in chronic lymphocytic leukemia cells is increased with the presence of interleukin 4 (IL-4), the hENT1-dependent uridine uptake remained unchanged [60]. Another study using a murine model of chronic myelogenous leukemia showed a decreased ENT1 activity reduction following reduced mRNA level of ENT1 [61]. Therefore, the transcription of hENT1 may not perfectly correlated with the uridine uptake activity, dual targeting hENT1 transcription and transporter activity might provide the utmost efficacy in gemcitabine utilization. Thus, a protein interaction screening strategy is needed to determine whether HNF4 $\alpha$ is involved in epigenetic regulation or posttranslational modification of hENT1.

\section{Conclusions}

In conclusion, we demonstrated that HNF4 $\alpha$ functioned as a tumor promoter gene that was upregulated in pancreatic cancer. Moreover, we discovered that higher expression of $\mathrm{HNF} 4 \alpha$ is correlated with poorer prognosis in PDAC patients. HNF4 $\alpha$ promotes pancreatic cancer cell proliferation and reduces gemcitabine-induced cell apoptosis. Mechanistically, we found that HNF4 $\alpha$ could downregulate hENT1, a membrane transporter of gemcitabine, at the transcriptional level. Taken together, these findings suggest that HNF4 $\alpha$ may serve as a potential novel predictive and therapeutic target for pancreatic cancer.

\section{Additional files}

Additional file 1: Table S1. HNF4a expression between PDAC and adjacent tissue in tumor tissue microarray.

Additional file 2: Figure S1. hENT1 expression is a prognostic factor in PDAC. a Representative images of IHC staining for hENT1 in tissue microarrays (scale bar, $200 \mu \mathrm{m}$; inset scale bar, $50 \mu \mathrm{m}$ ). b The overall survival of 
patients with PDAC was analyzed using the Kaplan-Meier analysis on the basis of hENT1 expression $\left(n=105,{ }^{*} P=0.0208\right)$.

Additional file 3: Figure S2. Correlation between the expression of HNF4a and dCK. Correlation analysis of HNF4a expression and dCK expression in PDAC tissues, as determined by the IHC score $(n=30, P=0.4281)$.

\section{Abbreviations}

BCl-2: B cell lymphoma 2; ChIP: chromatin immunoprecipitation; dCK: deoxycytidine kinase; DR1: direct repeat 1; FBS: foetal bovine serum; hENT1: human equilibrative nucleoside transporter 1; HNF4a: hepatocyte nuclear factor $4 a$; IHC: immunohistochemical; IL-4: interleukin 4; NR: nuclear receptor; PDAC: pancreatic adenocarcinoma; PARP1: poly ADP-ribose polymerase-1; qRT-PCR: quantitative real-time PCR; RR: ribonucleotide reductase; TNM: tumor node metastasis.

\section{Authors' contributions}

SRJ, XJY, XWX and YQ designed the experiments; QQS, WYX, QSH, and ZZ conducted the experiments; SRJ, XJY and XWX provided research materials and methods; QQS, SRJ and MQL analyzed data; and QQS and WYX wrote the manuscript. All authors read and approved the final manuscript.

\section{Author details}

${ }^{1}$ Department of Pancreatic Surgery, Fudan University Shanghai Cancer Center, Shanghai 200032, China. ${ }^{2}$ Department of Oncology, Shanghai Medical College, Fudan University, Shanghai 200032, China. ${ }^{3}$ Pancreatic Cancer Institute, Fudan University, Shanghai 200032, China. ${ }^{4}$ Shanghai Pancreatic Cancer Institute, Shanghai 200032, China.

\section{Acknowledgements}

Not applicable.

\section{Competing interests}

The authors declare that they have no competing interests.

\section{Availability of data and materials}

The datasets used in this study are available from the corresponding author upon reasonable request.

\section{Consent for publication}

Not applicable.

\section{Ethics approval and consent to participate}

This study was approved by the Ethics Committee of Fudan University Shanghai Cancer Centre (Shanghai, China).

\section{Funding}

This work was supported by the National Natural Science Foundation (81502031 and 81602085); the National Science Fund for Distinguished Young Scholars (81625016); and the Shanghai Sailing Program (16YF1401800).

\section{Publisher's Note}

Springer Nature remains neutral with regard to jurisdictional claims in published maps and institutional affiliations.

Received: 6 January 2019 Accepted: 28 February 2019

Published online: 04 March 2019

\section{References}

1. Hidalgo M. Pancreatic cancer. N Engl J Med. 2010;362:1605-17.

2. Siegel RL, Miller KD, Jemal A. Cancer statistics, 2018. CA Cancer J Clin. 2018;68:7-30

3. Zhang Y, Yang C, Cheng H, Fan Z, Huang Q, Lu Y, Fan K, Luo G, Jin K, Wang $Z$, et al. Novel agents for pancreatic ductal adenocarcinoma: emerging therapeutics and future directions. J Hematol Oncol. 2018;11:14.

4. Borowa-Mazgaj B. Pancreatic cancer-mechanisms of chemoresistance. Postepy Hig Med Dosw (Online). 2016;70:169-79.
5. Nammo T, Yamagata K, Tanaka T, Kodama T, Sladek FM, Fukui K, Katsube F, Sato Y, Miyagawa J, Shimomura I. Expression of HNF-4alpha (MODY1), HNF-1 beta (MODY5), and HNF-1alpha (MODY3) proteins in the developing mouse pancreas. Gene Expr Patterns. 2008;8:96-106.

6. Sladek FM, Zhong WM, Lai E, Darnell JE Jr. Liver-enriched transcription factor HNF-4 is a novel member of the steroid hormone receptor superfamily. Genes Dev. 1990;4:2353-65.

7. Hayhurst GP, Lee YH, Lambert G, Ward JM, Gonzalez FJ. Hepatocyte nuclear factor 4alpha (nuclear receptor 2A1) is essential for maintenance of hepatic gene expression and lipid homeostasis. Mol Cell Biol. 2001;21:1393-403.

8. Gonzalez FJ. Regulation of hepatocyte nuclear factor 4 alpha-mediated transcription. Drug Metab Pharmacokinet. 2008;23:2-7.

9. Darsigny M, Babeu JP, Seidman EG, Gendron FP, Levy E, Carrier J, Perreault $\mathrm{N}$, Boudreau F. Hepatocyte nuclear factor-4alpha promotes gut neoplasia in mice and protects against the production of reactive oxygen species. Cancer Res. 2010;70:9423-33.

10. Saandi T, Baraille F, Derbalwolfrom L, Cattin AL, Benahmed F, Martin E, Cardot P, Duclos B, Ribeiro A, Freund JN. Regulation of the tumor suppressor homeogene $\mathrm{Cd} \times 2$ by HNF4a in intestinal cancer. Oncogene. 2013;32:3782-8.

11. Ma Y, Wei $X$, Wu Z. HNF-4alpha promotes multidrug resistance of gastric cancer cells through the modulation of cell apoptosis. Oncol Lett. 2017;14:6477-84.

12. Mackey JR, Mani RS, Selner M, Mowles D, Young JD, Belt JA, Crawford CR, Cass CE. Functional nucleoside transporters are required for gemcitabine influx and manifestation of toxicity in cancer cell lines. Cancer Res. 1998;58:4349-57.

13. Spratlin J, Sangha R, Glubrecht D, Dabbagh L, Young JD, Dumontet C, Cass C, Lai R, Mackey JR. The absence of human equilibrative nucleoside transporter 1 is associated with reduced survival in patients with gemcitabine-treated pancreas adenocarcinoma. Clin Cancer Res. 2004;10:6956-61.

14. Bouffard DY, Laliberté J, Momparler RL. Kinetic studies on 2',2'-difluorodeoxycytidine (gemcitabine) with purified human deoxycytidine kinase and cytidine deaminase. Biochem Pharmacol. 1993;45:1857.

15. Hatzis P, Al-Madhoon AS, Jullig M, Petrakis TG, Eriksson S, Talianidis I. The intracellular localization of deoxycytidine kinase. J Biol Chem. 1998;273:30239-43.

16. Huang P, Chubb S, Hertel LW, Grindey GB, Plunkett W. Action of 2',2'-difluorodeoxycytidine on DNA synthesis. Cancer Res. 1991;51:6110-7.

17. Duxbury MS, Ito H, Zinner MJ, Ashley SW, Whang EE. RNA interference targeting the M2 subunit of ribonucleotide reductase enhances pancreatic adenocarcinoma chemosensitivity to gemcitabine. Oncogene. 2004;23:1539-48.

18. Bergman AM, Eijk PP, van Ruiz Haperen VW, Smid K, Veerman G, Hubeek I, van den ljssel P, Ylstra B, Peters GJ. In vivo induction of resistance to gemcitabine results in increased expression of ribonucleotide reductase subunit M1 as the major determinant. Cancer Res. 2005;65:9510-6.

19. de Sousa Cavalcante L, Monteiro G. Gemcitabine: metabolism and molecular mechanisms of action, sensitivity and chemoresistance in pancreatic cancer. Eur J Pharmacol. 2014;741:8-16.

20. Sierzega M, Pach R, Kulig P, Legutko J, Kulig J. Prognostic implications of expression profiling for gemcitabine-related Genes (hENT1, dCK, RRM1, RRM2) in patients with resectable pancreatic adenocarcinoma receiving adjuvant chemotherapy. Pancreas. 2017;46:684-9.

21. Marechal R, Bachet JB, Mackey JR, Dalban C, Demetter P, Graham K, Couvelard A, Svrcek M, Bardier-Dupas A, Hammel P, et al. Levels of gemcitabine transport and metabolism proteins predict survival times of patients treated with gemcitabine for pancreatic adenocarcinoma. Gastroenterology. 2012;143(664-674):e666.

22. Hu Q, Qin Y, Xiang J, Liu W, Xu W, Sun Q, Ji S, Liu J, Zhang Z, Ni Q, et al. dCK negatively regulates the NRF2/ARE axis and ROS production in pancreatic cancer. Cell Prolif. 2018;51:e12456.

23. Takano K, Hasegawa G, Jiang S, Kurosaki I, Hatakeyama K, Iwanari H, Tanaka T, Hamakubo T, Kodama T, Naito M. Immunohistochemical staining for P1 and P2 promoter-driven hepatocyte nuclear factor-4alpha may complement mucin phenotype of differentiated-type early gastric carcinoma. Pathol Int. 2009;59:462-70. 
24. Hu Q, Qin Y, Zhang B, Liang C, Ji S, Shi S, Xu W, Xiang J, Liang D, Ni Q, et al. FBW7 increases the chemosensitivity of pancreatic cancer cells to gemcitabine through upregulation of ENT1. Oncol Rep. 2017;38:2069-77.

25. Moffat J, Grueneberg DA, Yang X, Kim SY, Kloepfer AM, Hinkle G, Piqani B, Eisenhaure TM, Luo B, Grenier JK, et al. A lentiviral RNAi library for human and mouse genes applied to an arrayed viral high-content screen. Cell. 2006;124:1283-98.

26. Qin Y, Zhu W, Xu W, Zhang B, Shi S, Ji S, Liu J, Long J, Liu C, Liu L, et al. LSD1 sustains pancreatic cancer growth via maintaining HIF1alphadependent glycolytic process. Cancer Lett. 2014;347:225-32.

27. Abukhdeir AM, Park BH. P21 and p27: roles in carcinogenesis and drug resistance. Expert Rev Mol Med. 2008;10:e19.

28. Schmidt M, Fan Z. Protection against chemotherapy-induced cytotoxicity by cyclin-dependent kinase inhibitors (CKI) in CKI-responsive cells compared with CKI-unresponsive cells. Oncogene. 2001;20:6164-71.

29. Liu Z, Li D, Zheng X, Wang E, Wang J. Selective induction of apoptosis: promising therapy in pancreatic cancer. Curr Pharm Des. 2013;19:2259-68.

30. Brennan GT, Relias V, Saif MW. BRCA and pancreatic cancer. JOP. 2013:14:325-8.

31. Kim B, Srivastava SK, Kim SH. Caspase-9 as a therapeutic target for treating cancer. Expert Opin Ther Targets. 2015;19:113-27.

32. Ohhashi S, Ohuchida K, Mizumoto K, Fujita H, Egami T, Yu J, Toma H, Sadatomi S, Nagai E, Tanaka M. Down-regulation of deoxycytidine kinase enhances acquired resistance to gemcitabine in pancreatic cancer. Anticancer Res. 2008;28:2205-12.

33. Kim R, Tan A, Lai KK, Jiang J, Wang Y, Rybicki LA, Liu X. Prognostic roles of human equilibrative transporter 1 (hENT-1) and ribonucleoside reductase subunit M1 (RRM1) in resected pancreatic cancer. Cancer. 2011;117:3126-34.

34. Von Hoff DD, Ervin T, Arena FP, Chiorean EG, Infante J, Moore M, Seay T, Tjulandin SA, Ma WW, Saleh MN, et al. Increased survival in pancreatic cancer with nab-paclitaxel plus gemcitabine. N Engl J Med. 2013;369:1691-703

35. Wang Z, Benoit G, Liu J, Prasad S, Aarnisalo P, Liu X, Xu H, Walker NP, Perlmann T. Structure and function of Nurr1 identifies a class of ligandindependent nuclear receptors. Nature. 2003;423:555-60.

36. Walesky C, Apte U. Role of hepatocyte nuclear factor 4alpha (HNF4alpha) in cell proliferation and cancer. Gene Expr. 2015;16:101-8.

37. Safe S, Jin UH, Hedrick E, Reeder A, Lee SO. Minireview: role of orphan nuclear receptors in cancer and potential as drug targets. Mol Endocrinol. 2014:28:157-72.

38. Balk SP, Knudsen KE. AR, the cell cycle, and prostate cancer. Nucl Recept Signal. 2008;6:e001.

39. Hartman J, Strom A, Gustafsson JA. Estrogen receptor beta in breast cancer-diagnostic and therapeutic implications. Steroids. 2009;74:635-41.

40. Mueller E, Smith M, Sarraf P, Kroll T, Aiyer A, Kaufman DS, Oh W, Demetri G, Figg WD, Zhou XP, et al. Effects of ligand activation of peroxisome proliferator-activated receptor gamma in human prostate cancer. Proc Natl Acad Sci USA. 2000;97:10990-5.

41. Bian XL, Chen HZ, Yang PB, Li YP, Zhang FN, Zhang JY, Wang WJ, Zhao WX, Zhang S, Chen QT, et al. Nur77 suppresses hepatocellular carcinoma via switching glucose metabolism toward gluconeogenesis through attenuating phosphoenolpyruvate carboxykinase sumoylation. Nat Commun. 2017:8:14420.

42. Lee SO, Jin UH, Kang JH, Kim SB, Guthrie AS, Sreevalsan S, Lee JS, Safe S. The orphan nuclear receptor NR4A1 (Nur77) regulates oxidative and endoplasmic reticulum stress in pancreatic cancer cells. Mol Cancer Res. 2014;12:527-38.

43. Holbeck S, Chang J, Best AM, Bookout AL, Mangelsdorf DJ, Martinez ED. Expression profiling of nuclear receptors in the NCI60 cancer cell panel reveals receptor-drug and receptor-gene interactions. Mol Endocrinol. 2010;24:1287-96.

44. Bailey JM, Swanson BJ, Hamada T, Eggers JP, Singh PK, Caffery T, Ouellette MM, Hollingsworth MA. Sonic hedgehog promotes desmoplasia in pancreatic cancer. Clin Cancer Res. 2008;14:5995-6004.
45. DuFort CC, DelGiorno KE, Carlson MA, Osgood RJ, Zhao C, Huang Z, Thompson CB, Connor RJ, Thanos CD, Scott Brockenbrough J, et al. interstitial pressure in pancreatic ductal adenocarcinoma is dominated by a gel-fluid phase. Biophys J. 2016;1 10:2106-19.

46. Arlt A, Gehrz A, Müerköster S, Vorndamm J, Kruse M-L, Fölsch UR, Schäfer H. Role of NF-KB and Akt/PI3K in the resistance of pancreatic carcinoma cell lines against gemcitabine-induced cell death. Oncogene. 2003;22:3243

47. Semenza GL. Hypoxia-inducible factors: mediators of cancer progression and targets for cancer therapy. Trends Pharmacol Sci. 2012;33:207-14.

48. Skrypek N, Duchene B, Hebbar M, Leteurtre E, van Seuningen I, Jonckheere N. The MUC4 mucin mediates gemcitabine resistance of human pancreatic cancer cells via the Concentrative Nucleoside Transporter family. Oncogene. 2013;32:1714-23.

49. Meng Q, Shi S, Liang C, Liang D, Hua J, Zhang B, Xu J, Yu X. Abrogation of glutathione peroxidase-1 drives EMT and chemoresistance in pancreatic cancer by activating ROS-mediated Akt/GSK3beta/Snail signaling. Oncogene. 2018;37:5843-57.

50. Nordh S, Ansari D, Andersson R. hENT1 expression is predictive of gemcitabine outcome in pancreatic cancer: a systematic review. World J Gastroenterol. 2014;20:8482-90.

51. Greenhalf W, Ghaneh P, Neoptolemos JP, Palmer DH, Cox TF, Lamb RF, Garner E, Campbell F, Mackey JR, Costello E, et al. Pancreatic cancer hENT1 expression and survival from gemcitabine in patients from the ESPAC-3 trial. J Natl Cancer Inst. 2014;106:djt3347.

52. Yabushita Y, Mori R, Taniguchi K, Matsuyama R, Kumamoto T, Sakamaki K, Kubota K, Endo I. Combined Analyses of hENT1, TS, and DPD Predict Outcomes of Borderline-resectable Pancreatic Cancer. Anticancer Res. 2017:37:2465-76.

53. Pandolfi A, Di Pietro N. High glucose, nitric oxide, and adenosine: a vicious circle in chronic hyperglycaemia? Cardiovasc Res. 2010;86:9-11.

54. Hesler RA, Huang JJ, Starr MD, Treboschi VM, Bernanke AG, Nixon AB, McCall SJ, White RR, Blobe GC. TGF-beta-induced stromal CYR61 promotes resistance to gemcitabine in pancreatic ductal adenocarcinoma through downregulation of the nucleoside transporters hENT1 and hCNT3. Carcinogenesis. 2016;37:1041-51.

55. Wang JC, Stafford JM, Granner DK. SRC-1 and GRIP1 coactivate transcription with hepatocyte nuclear factor 4. J Biol Chem. 1998;273:30847-50.

56. Yoon JC, Puigserver P, Chen G, Donovan J, Wu Z, Rhee J, Adelmant G, Stafford J, Kahn CR, Granner DK, et al. Control of hepatic gluconeogenesis through the transcriptional coactivator PGC-1. Nature. 2001;413:131-8.

57. Candelaria M, de la Cruz-Hernandez E, Taja-Chayeb L, Perez-Cardenas E, Trejo-Becerril C, Gonzalez-Fierro A, Chavez-Blanco A, Soto-Reyes E, Dominguez G, Trujillo JE, et al. DNA methylation-independent reversion of gemcitabine resistance by hydralazine in cervical cancer cells. PLoS ONE. 2012;7:e29181.

58. Englert NA, Luo G, Goldstein JA, Surapureddi S. Epigenetic modification of histone 3 lysine 27: mediator subunit MED25 is required for the dissociation of polycomb repressive complex 2 from the promoter of cytochrome P450 2C9. J Biol Chem. 2015;290:2264-78.

59. Barrero MJ, Malik S. Two functional modes of a nuclear receptor-recruited arginine methyltransferase in transcriptional activation. Mol Cell. 2006;24:233-43.

60. Fernandez Calotti P, Galmarini CM, Canones C, Gamberale R, Saenz D, Avalos JS, Chianelli M, Rosenstein R, Giordano M. Modulation of the human equilibrative nucleoside transporter1 (hENT1) activity by IL-4 and PMA in B cells from chronic lymphocytic leukemia. Biochem Pharmacol. 2008;75:857-65.

61. Leisewitz AV, Zimmerman El, Huang M, Jones SZ, Yang J, Graves LM. Regulation of ENT1 expression and ENT1-dependent nucleoside transport by c-Jun N-terminal kinase. Biochem Biophys Res Commun. 2011;404:370-5. 